\title{
In Situ Quantification of Genomic Instability in Breast Cancer Progression
}

\author{
C. Ortiz de Solórzano ${ }^{1}$, K. Chin ${ }^{2}$, J.W. Gray ${ }^{2}$, S.J. Lockett ${ }^{3}$ \\ ${ }^{1}$ Life Sciences Division, Lawrence Berkeley National Laboratory, Berkeley, CA, USA \\ ${ }^{2}$ Department of Laboratory Medicine and Cancer Center, University of California, San Francisco, CA, USA \\ ${ }^{3}$ SAIC-Frederick, Frederick, MD, USA
}

\begin{abstract}
Genomic instability is a hallmark of breast and other solid cancers. Presumably caused by critical telomere reduction, GI is responsible for providing the genetic diversity required in the multi-step progression of the disease. We have used multicolor fluorescence in situ hybridization and 3D image analysis to quantify genomic instability cell-by-cell in thick, intact tissue sections of normal breast epithelium, preneoplastic lesions (usual ductal hyperplasia), ductal carcinona is situ or invasive carcinoma of the breast.. Our in situ -cell by cell- analysis of genomic instability shows an important increase of genomic instability in the transition from hyperplasia to in situ carcinoma, followed by a reduction of instability in invasive carcinoma. This pattern suggests that the transition from hyperplasia to in situ carcinoma corresponds to telomere crisis and invasive carcinoma is a consequence of telomerase reactivation afer telomere crisis.
\end{abstract}

Keywords - 3D image segmentation, genomic instability telomere crisis, FISH

\section{INTRODUCTION}

Genetic instability (GI) is believed to be an early event in breast cancer progression. GI is most likely caused by reduction of the chromosome ends (telomeres) beyond a critical size, in the absence of active telomerase. Cells containing critically short telomeres are prone to suffer chromosomal aberrations during mitosis, due to incorrect chromosome segregation. These chromosomal aberrations, perpetuated in cells that manage to survive by bypassing the cell cycle check points or by inactivating the apoptosis process, produce the "genetic diversity" that allows the accumulation of genetic abnormalities that drives the progression of the disease as the tissue evolves from normal to usual ductal hyperplesia (UDH) to ductal carcinoma in situ (DCIS) and finally to invasive carcinoma (IC) $[1,2]$.

We are interested in locating the onset of GI within the progression of the disease, and confirm the hypothesis that that GI is concomitant with telomere critical reduction.

GI can be studied by looking at gene copy variation across the entire genome (e.g. DNA microarrays). Useful as this approach is to obtain an average value of genomic instability, it obliterates tissue heterogeneity: since tumors are composed of different cell types and coexist with normal and preneoplastic epithelium and with normal fibroblasts, adipocites, lymphocytes, etc., it is difficult, if not impossible, to isolate areas containing a significant number of cells of a given type, as would be required when looking at the genomic changes of each tissue type. A different approach involves isolating, enriching and clonally expanding cells extracted from areas of the tumor. The cells can then be arrested at metaphase and multiple-color fluorescence in situ hybridization (MFISH) used to detect chromosomal rearrangements caused by GI. In this case, besides the technical problems related to cell culture, it is unclear how close the selected cells represent the tissue they come from.

To overcome those problems, we used two-color FISH, targeting two different regions of the genome (ctr.1, 20q13) and did a cell-by-cell enumeration of the copies of each target on 3D confocal images of selected tissue areas of NB, UDH, DCIS and IC. The tissue blocks were cut at 30 microns to ensure the presence of a sufficient number of intact nuclei. Then we created 3D histograms with the number of nuclei having each possible combination of the probes and determined -based on the width of the histogram- the level of instability of each type of tissue. This approach respects heterogeneity, since cells of only one type are used at a time. Furthermore, the cells are analyzed in their native tissue context.

Manually enumerating FISH signals in 3D confocal stacks is a cumbersome task, only feasible for very a small number of nuclei, and even in that case, very much subject to inter-observer variability. To analyze a highly variable phenomenon such as GI, a high number of nuclei must be analyzed in order to produce statistically significant data.

To solve this problem, we have developed 3D image analysis tools for nuclei and FISH signal segmentation in 3D confocal stacks. In this paper we present these methods along with the results obtained when comparing the levels of GI in breast cancer progression.

\section{Methodology}

\section{A. Tissue processing and Microscopy}

Frozen tissue blocks were obtained from the UCSF Breast Oncology Tissue Repository. Pathological evaluation of the tissue was performed on thin tissue sections cut from the top of the block and stained with Hematoxylin and Eosin (H\&E). Tissue areas were selected containing normal breast epithelium (NB), usual ductal hyperplasia (UDH), ductal carcinoma in situ (DCIS) or invasive carcinoma. 
Fig. 1. (LEFT) Nuclear and FISH segmentation

A) 3D Surface rendering of an area of normal epithelium from a YOPRO-1 counterstained block. Each nucleus is an individual entity (see blue selected nucleus) with morphological and positional parameters obtained using the nuclear segmentation algorithm described in the text. B) Cell by cell segmentation of two DNA probes: ctr.1 (red) and 20q13 (light blue). The signals were detected within the volume defined by each nucleus and rendered within the reconstruction of the tissue shown in A. C) close up on one of the nucleus from image B. D) Histogram showing the number of nuclei containing every possible combination of number of probes.

Adjacent thick $(20-40 \mu \mathrm{m})$ sections where cut, counterstained with YOPRO-1 and hybridized with a Cy5 tagged alpha-satellite probe (pUC1.77) and an Alexa568 labeled 20q13 probe.

All areas selected in the H\&E sections were identified in the contiguous thick sections and imaged using a confocal microscope (Zeiss LSM410), using a 63X, 1.3NA planapochromatic lense. Three consecutive scans were done per image with the 488, 568 and $633 \mathrm{~nm}$ laser lines, corresponding to YOPRO-1, Alexa568 and Cy5 respectively. The images had typically $512 \times 512 \times 100$ voxels, with a resolution of $0.2 \times 0.2 \times 03 \mu \mathrm{m}(\mathrm{X}, \mathrm{Y}, \mathrm{Z})$ which correspond to the theoretical resolution of the optical system, for the particular lens used.

\section{B. Image analysis}

The 3D images of the YOPRO-1 counterstained nuclei were segmented as described in [3]. Briefly, a gradientweighted adaptive threshold was used to separate nuclear volumes from the unstained background. Then each individual segmented object was rendered from the contours of the binary masks of the segmented objects using the MarchingCubes algorithm. Rendered versions of the segmented objects were presented to the user for classification using DaVinci, an in-house visualization program (Fig 1A). All objects were classified as single nuclei, clusters, debris or nuclei on the edge. Objects classified as cluster were further segmented by using a Watershed algorithm [4] applied on the Hough Transform of the cluster. The results of the segmentation were presented to the user for classification and, when necessary due to oversegmentation, for interactive joining.

FISH signals from ctr. 1 and $20 \mathrm{q} 13$ probes within each segmented nucleus were as described in [5]. Namely, we applied a Top Hat algorithm [6] to the corresponding fluorescent channels, but only within the extent of the nucleus, as defined by the nuclear segmentation algorithm. Gray-scale reconstruction [7] was built into the Top Hat to correct for distortion in the shape of the signals due to the non-ideal structuring element used in the Top Hat. Gaussian filtering was used after the Top Hat to eliminate noise. The signals were rendered in 3D using MarchingCubes, and incorporated into the $3 \mathrm{D}$ reconstruction of the tissue (Fig. 1B,C). Finally, bivariate frequency histograms were created with the ctr. 1 and 20q13 copy numbers.

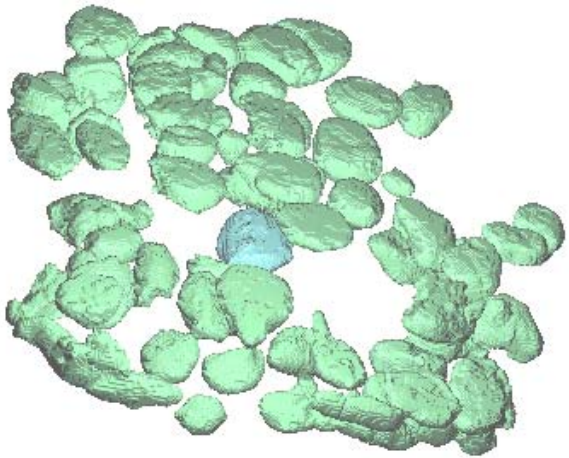

A
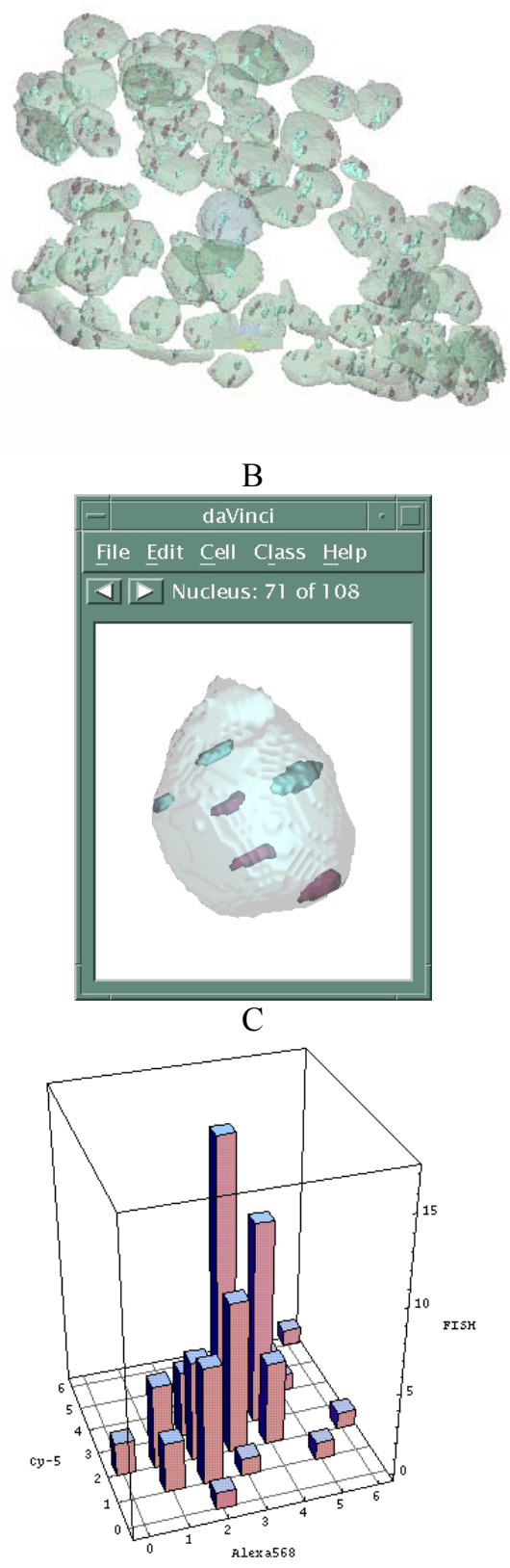

$\mathrm{D}$ 


\section{Error assesment}

The accuracy of the enumeration was assessed by using control areas of normal human skin and normal breast epithelium. The overall analytical accuracy was established by fitting a statistical model that assumed random loss of true signals and random detection of spurious signals to the bivariate histograms of all normal areas (a total of 537 nuclei).

\section{Correlation of nuclear volume and copy number}

Linear regression analysis was employed to find the correlation between copy number of ctr.1 and 20q13 and between the total number of signals (both ctr.1 and 20q13) and nuclear volume.

\section{RESULTS}

The statistical analysis done to determine the accuracy of the FISH segmentation algorithm indicated that $93 \pm 4 \%$ of genuine FISH signals were correctly detected, with a $4 \pm 4 \%$ probability of a spurious signal being incorrectly detected. These are satisfactory results, similar to those reported for disaggregated nuclei, thus confirming that our segmentation method is accurate enough for the analysis of copy number changes affecting more than a few percent of the cells of the population.

The level of instability in the progression NB-UDHDCIS-IC was calculated as the standard deviation of the bivariate copy number distribution calculated in several images of each type of tissue (one normal breast, one case of UDH, three cases of DCIS and two cases of IC).

Analysis of the UDH sample (data not shown) presented a unimodal bivariant distribution which however, contained a significant number of randomly distributed nuclei $(22 \%$, $\mathrm{p}<0.001)$ with a single copy of chromosome. All three DCIS cases shown an extremely high level of instability, one case showing correlation between copy number (ctr.1 plus 20q13) and nuclear volume, suggesting concomitant poliploidy. The two other DCIS cases did not show that correlation. Spatial statistical analysis of all three samples showed that nuclei with significantly different chromosomal composition were randomly mixed, suggesting that the genome copy variation was caused by genetic instability and not by clonal expansion. Analysis of IC showed high levels of instability in all cases, although slightly lover than in the DCIS specimens, with areas of both normal and abnormal ploidy. Cells were again randomly mixed, suggesting genuine instability and not clonal expansion. Figure 2 summarizes de results.

\section{DISCUSSION}

Our results (Figure2) show a significant increase in the level of instability as cells progress from pre-neoplasia (UDH) to DCIS, which although confined intraductally, it is composed of cells with a malignant phenotype. Instability slightly decreases in IC, suggesting that IC is composed of a clonal subpopulation of DCIS cells with a proliferative advantage. This suggests that critical telomere shortening is the cause of genomic instability, and that in tissue it happens in the transition from UDH to DCIS. IC would be formed by cells that "survive" telomeric crisis and are stabilized by telomerase reactivation or an alternative immortalization mechanism.

\section{CONCLUSION}

In this paper we have shown how FISH in thick tissue sections combined with 3D image analysis tools can be effectively used to study genomic instability in vitro. By using semi-automatic segmentation of nuclei from confocal images followed by automatic enumeration of FISH signals, we were able to estimate genetic instability by looking at the cell-by-cell accumulation of chromosomal abnormalities in intact tissue samples. Our cell-by-cell approach fits the highly heterogeneous nature of this phenomenon, which would have been undermined by other "bulk" methods, such as Southern blot analysis or MFISH.

As future developments, we are working on quantitative image-based methods to calculate telomere length in thick tissue samples using PNA probes. This would allow us to quantify both genomic instability and telomere length, thus providing an even better confirmation of our hypothesis that the transition to DCIS in fact coincides with telomere crisis.

\section{ACKNOWLEDGMENT}

This work was supported by Carl Zeiss Inc, Vysis, Inc., the Director, Office of Energy Research, Office of Health and Environmental Research of the U.S. Department of Energy under contract NO. DE-AC03-76SF00098; NIH contract NO. N01-CO-56000, NIH grants CA67412 and CA58207, the Avon foundation, and the Department of Defense Breast Cancer Research Program, grants DAMD1700-1-0227 and DAMD17-00-1-0306. We thank Drs. Daniel Pinkel and Donna Albertson for helpful discussions and advice, Dr. Curtis Thompson for his advice concerning the thick section FISH, Mr. Ramin Khavari for labeling the normal skin tissue, Ms Carmina Florendo for sectioning the tissue and Ms. Karen Chew for the histological evaluations. 


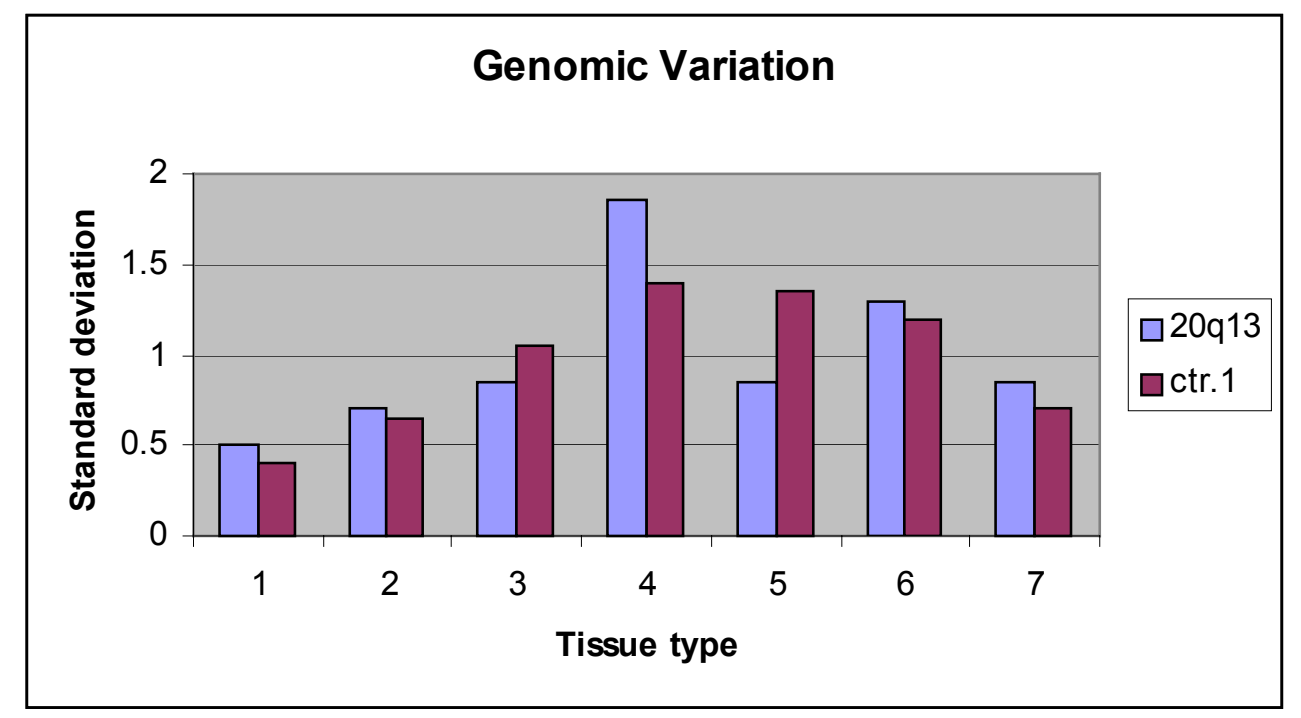

Fig. 2. Genomic Instability in Breast Cancer Progression

Standard deviation of the bivariate distributions of ctr.1 and 20q.13 in biopsied tissue.

Legend for X axis: 1) Normal; 2) ADH; 3,4,5) DCIS; 6\&7) IC

\section{REFERENCES}

[1] C. Lengauer, K.W. Kinzler \& B. Volgestein, "Genetic instabilities in human cancers" Nature., vol. 396, pp. 643-649, 1998.

[2] K.R. Loeb \& L.A. Loeb, "Significance of multiple mutations in cancer" Carcinogenesis., vol. 21, pp. 379-385, 2000.

[3] C. Ortiz de Solórzano, E. García Rodríguez, A. Jones., D. Pinkel, J.W. Gray, D. Sudar \& S.J. Lockett, "Segmentation of confocal microscope images of cell nuclei in thick tissue sections" Journal of Microscopy-Oxford, vol. 193, pp. 212-226, 1999.

[4] S. Beucher \& F. Meyer, The morphological approach to segmentation: The watershed transformation. Marcel Dekker Inc., NY, 1992, pp. 433-481.

[5] C. Ortiz de Solórzano, A. Santos, I. Vallcorba, J.M. GarcíaSagredo \& F. del Pozo, "Automated FISH spot counting in Interphase Nuclei: Statistical Validation and Data Correction" Cytometry, vol. 31, pp. 93-99, 1998.

[6] J. Serra, Image Analysis and Mathematical Morphology. Academic Press, NY, 1982, pp. 436-437.

[7] L. Vincent, "Morphological grayscale reconstruction in image analysis: Applications and efficient algorithms" IEEE Trans. on Image Proc., vol. 2, pp. 172-201, 1993.

[8] G. Nonet et al, "The ZNF217 gene amplified in breast cancers promotes immortalization of human mammary epithelial cells" Cancer Research., vol. 61, pp. 1250-1254, 2001. 